\title{
Современное состояние проблемы хирургического лечения неопухолевых заболеваний толстой кишки и факторы, влияющие на его результаты. Часть 1.
}

\author{
В. М. Мельник, А. И. Пойда, Абдулрахман Абдул Кадир
}

Национальный медицинский университет имени А. А. Богомольца, г. Киев

\section{Modern state of a problem of surgical treatment for nontumoral diseases of large bowel and factors, influencing its results. Part 1.}

\author{
V. M. Melnyk, A. I. Poyda, Abdulrahman Abdul Qadir \\ Bogomolets National Medical University, Kyiv
}

В течение последних 25 лет в большинстве экономически развитых стран мира, а также в Украине стремительно увеличивается частота неопухолевых заболеваний толстой кишки (ТК), а именно: язвенного колита, болезни Крона ТК, семейного аденоматозного полипоза [1]. За последние 40 лет число пациентов с тотальным или субтотальным поражением ТК, особенно при язвенном колите, увеличилось с 38 до 52\% [2]. В связи с этим прослеживается тенденция к увеличению числа операций, выполняемых на ТК, и на этом фоне к улучшению их результатов. Некоторые авторы сообщают о снижении частоты осложнений после операций на ТК с 16,2 - 32,6 до 4,3 - 6,1\%, а послеоперационной летальности с 3,7 - 14,3 до 0 - 3,5\% [3, 4]. Указанную положительную тенденцию объясняют широким внедрением современных диагностических и лечебных технологий в практику хирургии ТК, в частности, эндоректального ультразвукового исследования, компъютерной и магнитно-резонанской томографии, видео- и хромоколоноскопии, лапароскопических технологий, эндоректальных методов лечения, современных сшивающих аппаратов, дифференцированного подхода к выбору объема радикальных операций, совершенствованием способов выполнения восстановительных и реконструктивно-восстановительных операций $[1,3,4]$.

Важным фактором, способствующим улучшению результатов оперативных вмешательств на ТК, считают совершенствование лечебной тактики при язвенном колите и болезни Крона, в частности, признание консервативной терапии приоритетным методом лечения указанных заболеваний [5, 6]. Это стало возможным благодаря широкому применению современных препаратов для базисной терапии: производных месалазина, системных и топических глюкокортикоидов, антицитокинов [7, 8]. Достаточно эффективная терапия, применяемая при язвенном колите и болезни Крона, способствует увеличению периода ремиссии, уменьшению активности воспалительного процесса, агрессивности течения указанных заболеваний, ограничению показаний к выполнению хирургических вмешательств и, что особенно важно, значительному снижению частоты их выполнения по поводу острых осложнений: кровотечения, перфорации, перитонита, токсической дилатации ТК [5, 6]. Однако в связи с агрессивным течением 20 - 30\% больных с язвенным колитом и 75 - 90\% - с болезнью Крона на протяжении жизни нуждаются в хирургическом лечении $[9,10]$.

Чрезвычайно важное направление хирургии ТК - совершенствование методов прогнозирования и профилактики послеоперационных осложнений на этапе подготовки к предстоящей операции, ее выполнения, а также после операции $[3,4]$. Для успешной реализации указанного положения многие авторы в период подготовки к выполнению хирургического вмешательства, кроме мероприятий, направленных на улучшение функции жизнеобеспечивающих органов и систем, большое значение придают современной антибиотикопрофилактике, методам механической очистки ТК с использованием фортранса, эндофалька, пикопрепа, которые, способствуя очищению ТК, уменьшают риск возникновения гнойно-септических осложнений $[4,11]$.

Во время выполнения оперативного вмешательства, если есть показания, применяют декомпрессию ТК, оценивают состояние кровоснабжения ее отрезков, подлежащих соединению, достаточную их мобилизацию, проводят подготовку для наложения анастомоза, выбирают оптимальный способ его формирования и протекции [3, 4].

В послеоперационном периоде методы профилактики осложнений преимущественно направлены на коррекцию показателей гемодинамики, нормализацию перфузии тканей, функций свертывания крови, внешнего дыхания, почек, других органов и систем, микроциркуляции, улучшение перистальтики и пропульсии кишечника [4, 12].

Кроме указанных факторов, результаты операций на ТК в значительной степени зависят от особенностей выполнения хирургического вмешательства, преимущественно от выбора объема радикального, а также технических аспектов выполнения восстановительного или реконструктивно-восстановительного этапов. 
Выбор объема радикального этапа оперативного вмешательства зависит от протяженности поражения патологическим процессом анатомических отделов ТК, наличия осложнений основного заболевания, степени его выраженности, тяжести состояния больного, наличия тяжелых осложнений неопухолевых заболеваний ТК: перитонита, токсической дилатации ТК, кишечного кровотечения [2, 13, 14]. У таких больных преимущественно выполняют «лечебную» колэктомию, формируют культю прямой кишки, выводят концевую илеостому $[2,15,16]$. При болезни Крона, в зависимости от особенностей поражения ТК, отдают предпочтение сегментарным резекциям, учитывая высокий риск рецидива заболевания в оставшихся анатомических отделах кишечника и необходимость в связи этим выполнения повторных операций [17]. При субтотальном проксимальном поражении ТК болезнью Крона без видимых изменений слизистой оболочки прямой кишки или дистальных ее отделов некоторые авторы отдают предпочтение выполнению колэктомии или колэктомии с резекцией прямой кишки [10, 17]. При других тяжелых неопухолевых заболеваниях, в том числе при тотальном поражении болезнью Крона ТК, на радикальном этапе, по мнению большинства авторов, необходимо удаление всех анатомических отделов ТК, в которых возможна пролонгация или рецидив заболевания. Такими радикальными операциями в настоящее время являются колэктомия с низкой передней резекцией прямой кишки [10, 18], а также колэктомия с предельно низкой передней резекцией прямой кишки, мукозэктомией хирургического анального канала до зубчатой линии [19 - 21]. Мукозэктомия заключается в удалении слизистой оболочки хирургического анального канала, которая после колэктомии и предельно низкой передней резекции прямой кишки остается единственным участком, в котором возможна пролонгация или рецидив язвенного колита, болезни Крона ТК, семейного аденоматозного полипоза [22]. Поэтому необходимость выполнения мукозэктомии обусловлена стремлением обеспечить достаточный радикализм хирургического вмешательства, сохранить сфинктерный аппарат прямой кишки и, следовательно, функцию анального держания. Для решения указанной проблемы некоторые авторы достаточно обоснованно предлагают дифференцированный подход к выполнению мукозэктомии, который заключается в применении менее травматического метода, предусматривающего удаление только слизистой оболочки и сохранение подслизистой основы хирургического анального канала [4]. Метод показан при заболеваниях, когда не поражена подслизистая основа: семейном аденоматозном полипозе и язвенном колите (I, II степени активности воспалительного процесса) в нижнеампулярном отделе прямой кишки [4]. При болезни Крона, а также язвенном колите (III степень активности воспалительного процесса в области хирургического анального канала) - состояниях, при которых поражена подслизистая основа, авторы вынужденно выполняют мукозсубмукозэктомию, предусматривающую удаление слизистой оболочки и подслизистой основы, несмотря на большую травматичность манипуляции и риск повреждения внутреннего сфиктера прямой кишки [4]. Однако при минимальных изменениях слизистой оболочки (І степень активности воспалительного процесса) [23, 24] без полипов или дисплазии в дистальном участке прямой кишки $[10,25]$ некоторые хирурги выполняют колэктомию с низкой резекцией прямой кишки, целью которой является сохранение дистального участка прямой кишки, преиму- щественно на уровне анальных столбов и крипт, покрытого переходным эпителием кубического типа, протяженностью 2,5 - 3 см. Нижняя граница этого участка располагается на 0,6 - 1 см дистальнее, а верхняя - на 1,5 - 2 см проксимальнее зубчатой линии $[15,28]$. Этот участок анального канала содержит значительное количество чувствительных нервных окончаний, что играет важную роль в регуляции функции опорожнения $[1,15,25]$. Принято считать, что данный участок принимает участие в «распознавании» газа, жидкого и плотного кишечного содержимого. С учетом изложенного, а также в связи с тем, что воспалительные изменения в указанном участке обычно менее выражены по сравнению с проксимально расположенными участками прямой кишки, выбор радикальной операции, предусматривающей его сохранность, а именно колэктомии с низкой передней резекцией прямой кишки, по мнению некоторых авторов, обоснован и предпочтителен $[15,18]$. Сохранность дистального участка прямой кишки обеспечивает улучшение в определенной степени резервуарных свойств создаваемых тонкокишечных тазовых резервуарных конструкций, функции анального держания, опорожнения, сексуальной функции вследствие менышей травмы тазового нервного сплетения, функции внутренних половых органов, что особенно важно для пациентов молодого возраста [18, 20, 27]. Указанными преимуществами колэктомии с низкой передней резекцией прямой кишки обосновывают ее применение при язвенном колите $[18,23]$, семейном аденоматозном полипозе [20, 25, 27], а также, по мнению некоторых авторов, при тотальном поражении болезнью Крона ТК [4, 28, 29] у тех больных, у которых нет выраженных патологических изменений в дистальном участке слизистой оболочки прямой кишки [4, 20, 30].

Агрессивное течение язвенного колита, болезни Крона ТК, несоблюдение рекомендаций по применению противорецидивного лечения приводят к хроническому воспалительному процессу в участке прямой кишки, покрытом переходным эпителием, у 22 - 84,9\% больных [15, 30, 31]. В отношении сохраненного участка прямой кишки существует высокий риск рецидива семейного аденоматозного полипоза [25, 27]. Упорное течение воспалительного процесса вследствие рецидива заболевания приводит к дисплазии слизистой оболочки в области участка, покрытого переходным эпителием, у 18 - 42\% пациентов [15, 22, 32], более частому возникновению рака, риск которого увеличивается в зависимости от сроков наблюдения $[16,30]$ и при язвенном колите составляет 3,8 - 9\%; 6,9 - 20,1\%; 14,3 - 25,1\% через 10 , 15 и 20 лет [31, 33], при семейном аденоматозном полипозе - 3,8 - 4\%; 21,7 - 25\%; 23,9\% - через 5, 20, 25 лет соответственно [20]. Указанные неблагоприятные последствия колэктомии с низкой передней резекцией прямой кишки являются показанием к удалению ее оставшегося нижнеампулярного отдела, мукозэктомии хирургического анального канала и выполнению повторной реконструктивновосстановительной операции у 4,2 - 53\% [31] или экстирпации культи прямой кишки, резервуарэктомии, формированию пожизненной илеостомы у 8,9 - 53\% [34, 35] оперированных больных.

Поэтому большинство авторов отдают предпочтение выполнению первичных радикальных операций при указанных заболеваниях. Единственной достаточно радикальной операцией при язвенном колите, условно радикальной при семейном аденоматозном полипозе и болезни Крона 
ТК (с учетом возможного поражения указанными заболеваниями тонкой кишки) является колэктомия с предельно низкой передней резекцией прямой кишки, мукозэктомией хирургического анального канала до зубчатой линии $[10,36,37]$, позволяющая сохранить сфинктерный аппарат прямой кишки и функцию анального держания, следовательно, обеспечить условия для выполнения реконструктивно-восстановительного этапа [10, 36, 38]. Удельный вес указанной операции в структуре колопроктологических операций составляет 5,1 - 7,3\% [4, 30]. Она является основным методом хирургического лечения при язвенном колите у 42,1 - 90\% [5, 19, 39], семейном аденоматозном полипозе у 68,7 - 91\% [30, 40] больных. Ее достаточно часто выполняют при болезни Крона ТК - у 21,7 - 31,6\% больных [10, 41]. После этой операции не возникает рецидив язвенного колита, семейного аденоматозного полипоза в слизистой оболочке ТК, существенно уменьшается риск рецидива болезни Крона в связи с тем, что она включает удаление анатомических отделов ТК и участков слизистой оболочки, в которых возможно его возникновение [10, 20, 42].

После выполнения радикальных хирургических вмешательств на ТК при неопухолевых заболеваниях возникают тяжелые патологические состояния: диарейный и постколэктомический синдромы $[14,43]$. Понятием постколэктомического синдрома обозначают совокупность органических, функциональных и обменных нарушений со стороны пищеварительного канала, других органов и систем в виде преимущественных нарушений водно-электролитного, белково-энергетического обмена, функции печени и почек, возникающих после удаления ТК [3, 44, 45]. В условиях физиологического течения процессов пищеварения и всасывания на протяжении суток из тонкой кишки в толстую попадает в среднем 1000 мл химуса [4, 44]. В просвете ТК происходит дальнейшая его ферментативная обработка и всасывание ингредиентов, а именно воды, электролитов, микроэлементов, некоторых аминокислот, белков-ферментов [4, 44]. Удаление ТК приводит к утрате значительной площади всасывательной поверхности, что сопровождается нарушением сократительной функции стенки тонкой кишки, компенсаторным усилением в ней процессов всасывания. При этом в тонкой кишке преобладают ритмичные сегментарные сокращения, а не пропульсивные волны, что способствует компенсаторной задержке продвижения содержимого, уменьшению интенсивности процессов секреции, преобладанию процессов всасывания, сгущению химуса и подготовки его к эвакуации [44, 45]. Таким образом, оставшаяся тонкая кишка выполняет определенную часть функций удаленной ТК. Из-за повышенной функциональной нагрузки возникают необратимые морфологические изменения тонкой кишки. Макроскопически это проявляется гипотоническим состоянием ее стенки, на месте неглубоких перистых складок слизистой оболочки образуются «грубые» складки, участки гаустральноподобных сужений и округлых расширений, что свидетельствует о возникновении процесса колонизации [44, 45], а микроскопически отмечают атрофию большинства ворсинок слизистой оболочки, увеличение количества бокаловидных клеток, возникновение крипт, изменение бактериального спектра тонкокишечного содержимого, состав которого в значительной степени сходен с бактериальным спектром содержимого ТК [45]. По данным большинства авторов, указанные компенсаторные процессы, направленные на улуч- шение процессов всасывания, недостаточно эффективны и не обеспечивают физиологическую потребность и стабильность водно-электролитного и белково-энергетического баланса организма оперированного пациента вследствие выраженного диарейного синдрома, труднокомпенсированной потери воды, электролитов, микроэлементов, белков-ферментов. Именно поэтому у таких больных, кроме морфофункциональных нарушений со стороны оставшейся тонкой кишки, возникают тяжелые нарушения функции других органов и систем, в частности печени, почек, сердечно-сосудистой и дыхательной систем [44, 45].

Исследование функционального состояния гепатобилиарной системы при постколэктомическом синдроме с использованием рентгенорадионуклидного метода указывает на уменьшение поглотительной и выделительной функций печени, возникновение гипомоторной дискинезии внепеченочных желчных путей, что приводит к нарушению холединамики, всасывания жиров, возникновению желчнокаменной болезни, жировой дистрофии печени [44, 45]. При исследовании функции почек отмечают уменьшение их секреторной и экскреторной функций. Это сопровождается тенденцией к олигоурии, оксалатурии, возникновению мочекислого диатеза, мочекаменной болезни [3, 44].

Ухудшение состояния водно-электролитного и белково-энергетического баланса приводит к нарушению функций сердечно-сосудистой и дыхательной систем: тахикардии, нарушению сердечного ритма, гипотонии, дыхательной недостаточности. Патологические состояния, возникающие при постколэктомическом синдроме со стороны тонкой кишки, печени, почек, сердечно-сосудистой, дыхательной систем, требуют систематического консервативного лечения или выполнения повторного хирургического вмешательства, направленного на создание условий для улучшения процессов всасывания и коррекции указанных нарушений [3, 44]. Наиболее агрессивное течение диарейного и постколэктомического синдромов наблюдается после формирования илеостомы, прямых илеоректальных, илеоанальных анастомозов без резервуарных тонкокишечных конструкций. Это обусловлено сокращением времени транзита содержимого по тонкой кишке, уменьшением интенсивности процессов кишечного пищеварения, всасывания, значительной потерей химуса $[3,44,45]$.

Изложенное дает основания сделать такой вывод: при тяжелых неопухолевых заболеваниях ТК чрезвычайно важно выбрать оптимальный объем радикального этапа хирургического вмешательства и применить методы реконструктивно-восстановительных операций, способствующие улучшению функциональных результатов и обеспечивающие эффективную профилактику постколэктомического и диарейного синдромов. Технические и тактические аспекты указанных реконструктивно-восстановительных операций мы рассмотрим в следующем обзоре литературы.

\section{References}

1. Rivkin VL, Fayn SN, Bronshteyn AS, An VKM. Rukovodstvo po koloproktologii. Moskva: Medpraktika; 2004. 488 s. [In Russian].

2. Lorenzo G, Maurizio C, Maria LP, Tanzanu M, Silvio L, Mariangela P, et al. Ileal pouch-anal anastomosis 20 years later: is it still a good surgical option for patients with ulcerative colitis?. Int J Colorectal Dis. 2016 Dec; 31(12):1835-1843. doi: 10.1007/s00384-016-2657-8.

3. Melnyk VM. Rekonstruktyvno-vidnovni operatsii pry vydalenni funktsionalno-aktyvnykh viddiliv tovstoi kyshky. [thesis]. Kyiv. 2008. 41 s. [In Ukrainian]. 
4. Poyda AI, Mel'nik VM. Vosstanovitel'nye i rekonstruktivno-vosstanovitel'nye operatsii na tolstoy kishke. Kyiv: Izdatel'skiy tsentr «Imidzh Ukrainy»; 2014. 368 s. [In Russian].

5. Mozafar M, Shateri K, Tabatabaey A, Lotfollahzadeh S, Atqiaee K. Familial adenomatous polyposis: ileo-anal pouch versus ileo-rectal anastomosis. Gastroenterol Hepatol Bed Bench 2014 Fall;7(4): 206-10. PMID: 85874.

6. Leal RF, Ayrizono MLS, Milanski M, Fagundes JJ, Moraes JC, Meirelles LR, et al. Detection of epithelial apoptosis in pelvic ileal pouches for ulcerative colitis and familial adenomatous polyposis. J Transl Med. $2010 \mathrm{Jan}$ 29;8:11. doi: 10.1186/1479-5876-8-11

7. Bo Shen, Xiuli Liu. De Novo Collagenous Cuffitis. Inflamm Bowel Dis. 2011 February 2; 17(5):1249-50. Available from: https://doi.org/10.1002/ ibd. 21448.

8. Dolejs S, Kennedy G, Heise CP. Small bowel obstruction following restorative proctocolectomy: affected by a laparoscopic approach? J Surg Res. 2011 Oct; 170(2):202-8. doi: 10.1016/j.jss.2011.03.004.

9. Loftus EV Jr, Friedman HS, Delgado DJ, Sandborn WJ. Colectomy Subtypes, Follow-up Surgical Procedures, Postsurgical Complications, and Medical Charges Among Ulcerative Colitis Patients with Private Health Insurance in the United States. Inflamm Bowel Dis. 2009 Apr;15(4):56675. doi: 10.1002/ibd.20810.

10. Teixeira FV, Kelly KA. The physiology of intestinal pouches. Chirurg, 1999 May 01;70(5):513-9. doi: 10.1007/s001040050682.

11. Fursevich AM. Anatomicheskoe obosnovanie formirovaniya tazovogo tonkokishechnogo rezervuara posle proktokolektomii. Zdravookhranenie. 2000;(6):13-15. [In Russian].

12. Karyakina LI, Karyakin GS, Bashabaev MT. Khirurgicheskoe lechenie nespetsificheskikh kolitov za 23 goda. Aktual'nye voprosy koloproktologii. Materialy II sezda koloproktologov Rossii s mezhdunarodnym uchastiem. Ufa; 2007. 443-5. [In Russian].

13. Lauranne AAP Derikx, Wietske Kievit, Joost PH Drenth, Dirk J de Jong, Cyriel Y Ponsioen, Bas Oldenburg, et al. Prior colorectal neoplasia is associated with increased risk of ileoanal pouch neoplasia in patients with inflammatory bowel disease. Gastroenterology, 2014 Jan;146(1):119-28. doi: 10.1053/j.gastro.2013.09.047.

14. Rossi HL, Brand MI, Saclarides TJ. Anal complications after restorative proctocolectomy (J-pouch). Am Surg. 2002 Jul;68(7):628-30. PMID: 12132747.

15. Jowett SL, Seal CJ, Barton JR, Welfare MR. The short inflammatory bowel disease questionnaire is reliable and responsive to clinically important change in ulcerative colitis. Am J Gastroenterol. 2001 Oct;96(10):2921-8. doi: 10.1111/j.1572-0241.2001.04682.x.

16. Lingohr P, Galetin T, Matthaei H, Straub E, Jafari A, Bölke E, et al. Vestweber Malignant melanoma of the ileo-anal pouch anastomosis after restorative proctocolectomy for ulcerative colitis: report of a case. Eur J Med Res. 2013 Nov 4;18:39. doi: 10.1186/2047-783X-18-39.

17. Belyanskiy LS, Poyda AI, Melnik VM, Kucher ND, Krivoruk MI. Khirurgicheskaya taktika pri bolezni Krona. Klinichna khirurhiia. 2016;10. 3:478. [In Russian].

18. Herrera L. Familial Adenomatosis Polyposis. New York: Alan R. Liss Inc; 1990. 402 p.

19. GhossainiNA, Maunoury V, Delcenserie R, Bulois P. Endoscopic treatment of a completely obstructed ileorectal anastomosis. Endoscopy 2014 Dec 13;46:609-10. doi: 10.1055/s-0034-1390753.

20. Pina-Pintor M, Ares P, Bona R. Severe steroid unresponsive ulcerative colitis outcome of restorative proctcolectomy in ppatients undergoing cyclosporine treatement. Dis. Colon Rectum. 2000;43(7):609-13. https://doi. org/10.1007/BF02235571.

21. Kravchenko TH. Kachestvo zhyzny bolnыkh, operyrovannыkh po povodu yazvennoho kolyta. Liky Ukrainy. 2015;3(24):39-43. [In Ukrainian].

22. Hulten L, Willen R, Nilsson O. Mucosal assessment for dysplasia and cancer in the ileal pouch mucosa in patients operated on for ulcerative colitis a 30-year follow-up study. Dis Colon Rectum. 2002 Apr 1;45(3):448-52. doi: 10.1007/s10350-004-6218-9.

23. Gorgun E, Sapci I, Onder A, Ozuner G, Liska D, Stocchi L, et al. Factors associated with portomesenteric venous thrombosis after total colectomy with ileorectal anastomosis or end ileostomy. Am J Surg. 2018;215:62-5. doi: https://doi.org/10.1016/j.amjsurg.2017.10.002.

24. Lepistö A, Kärkkäinen P, Järvinen HJ. Prevalence of primary sclerosing cholangitis in ulcerative colitis patients undergoing proctocolectomy and ileal pouch-anal anastomosis. Inflamm Bowel Dis. 2008 Jun;14(6):775-9. doi: $10.1002 /$ ibd.20384.
25. Odaryuk TS, Vorob'ev GI, Shelygin YuA. Khirurgiya raka pryamoy kishki. Moskva: OOO «Dedalus»; 2005. 235 s. [In Russian].

26. Zárate CA, Zúñiga DA, Pinedo MG, López KF, Molina PME, Viviani GP. Ileal pouch-anal anastomosis for ulcerative colitis: complications and long term functional results. Rev Med Chil. 2008 Apr;136(4): 467-74. doi:/ S0034-98872008000400007.

27. Matheus Cde O, Waisberg J, Zewer MH, Godoy AC. Syndrome of duodenal compression by the superior mesenteric artery following restorative proctocolectomy: a case report and review of literature. Sao Paulo Med J. 2005 May 2;123(3):151-3. doi: /S1516-31802005000300013.

28. Banasiewicz T, Walkowiak J, Drzymała-Czyż S, Borejsza-Wysocki M, Majewski P, Biczysko M, et al. Stricture of ileo-anal J pouch anastomosis increased the intensity of pouchitis in an experimental rat model. Med Sci Monit, 2012 Oct;18(10):409-13. doi: 10.12659/MSM.883481.

29. Aygara M, Yetisira F, Salman E, Yıldırıma MB, Özdedeo glu M, Durak D, et al. Rectal eversion and double-stapled ileal pouch anal anastomosis infamilial adenomatous polyposis syndrome. International Journal of Surgery Case Reports. 2014;(5):806-10. doi; 10.1016/j.ijscr.2014.08.013.

30. Shen B. Crohn's Disease of the Ileal Pouch: Reality, Diagnosis, and Management. Inflamm Bowel Dis. 2009 Feb 1;15:284-94. doi.org: 10.1002/ ibd.20661

31. Scoglio D, Ali UA, Fichera A. Surgical treatment of ulcerative colitis: Ileorectal vs ileal pouch-anal anastomosis. World J Gastroenterol, 2014 Oct 7;20(37):13211-8. doi: 10.3748/wjg.v20.i37.13211.

32. van Westreenen HL, Visser A, Tanis PJ, Bemelman WA. Morbidity related to defunctioning ileostomy closure after ileal pouch-anal anastomosis and low colonic anastomosis. Int J Colorectal Dis. 2012 Jan;27(1):49-54 doi: $10.1007 / \mathrm{s} 00384-011$.

33. Leal RF, Ayrizono Mde L, Coy CS, Fagundes JJ, GГies JR. [Short-term and long-term postoperative complications after ileal pouch-anal anastomosis in familial adenomatous polyposis]. Arq Gastroenterol. $2008 \mathrm{Apr}-$ Jun;45(2):106-10. PMID: 18622462.

34. O'Riordan JM, O'Connor BI, Huang H, Victor JC, Grufe R, MacRae HM, et al. Long-term outcome of colectomy and ileorectal anastomosis for Crohn's colitis. Dis Colon Rectum. 2011 Nov;54(11):1347-54. doi: 10.1097/DCR. 0b013e31822c9b2d.

35. Ozuner G, Hull T, Lee P, Fazio VW. What Happens to a Pelvis Pouch When a Fistula Develops? Dis Colon Rectum. 1997 May;40(5):543-7. doi.org: 10.1007/BF02055375.

36. Rodolfo J. Oviedo, Bruce M. Dixon, Chase W. Sofiak. Emergency total proctocolectomy in an uninsured patient with Familial Adenomatous Polyposis Syndrome and acute lowergastrointestinal hemorrhage in a community hospital: A case report. International Journal of Surgery Case Reports. 2016 Jul 30;(26):166-9. doi: 10.1016/j.ijscr.2016.07.052

37. Sandier RS, Eisen GM. Epidemiology of inflammatory bowel disease: In inflammatory bowel disease Fifth edition. Sanders. 2000; P. 89-113.

38. Rintala RJ, Lindahl HG. Proctocolectomy and J-pouch ileo-anal anastomosis in children. J pediatr Surg. 2002 Jan;37(1):66-70. PMID: 11781989

39. Arrossi AV, Kariv Y, Bronner MP, Hammel J, Remzi FH, Fazio VW, Goldblum JR. Backwash ileitis does not affect pouch outcome in patients with ulcerative colitis with restorative proctocolectomy. Clin Gastroenterol Hepatol. 2011 Nov;9(11):981-8. doi: 10.1016/j.cgh.2011.07.018.

40. Shorthouse A. Complications in pelvic pouch surgery. In: XII central european congress of coloproctology. Moskva; 2008. 111-2 p.

41. Karpukhin OYu, Mozhanov EV, Kolomenskiy SN, Cherenkov EV, Gil'mutdinov AA, Slivochkina NS, Shakurov AF. K voprosu o lechenii bolezni Krona u vzroslykh. Koloproktologiya (prilozhenie). 2011;3(37):130. [In Russian].

42. Poyda AI, Mel'nik VM. Obosnovanie formirovaniya i rezul'taty primeneniya tazovoy tonkokishechnoy rezervuarnoy konstruktsii novogo tipa posle kolektomii, predel'no nizkoy peredney rezektsii pryamoy kishki, mukozektomii. Chast' 1. Klinicheskaya khirurgiya. 2016;(4):5-8. [In Russian].

43. Bo Shen, Xiuli Liu. De Novo Collagenous Cuffitis. Inflamm Bowel Dis. 2011 Fab 2;17(5):1249-50. doi: 10.1002/ibd.21448.

44. Baltaytis YuV. Obshirnye rezektsii tolstoy kishki. Kyiv: Zdoroviya. 1990. 175 s. [In Russian].

45. Zherlov GK, Bashirov SR. Rezervuarnyy ileorektal'nyy anastomoz. Khirurgiya. 2001;(9):35-40. [In Russian]. 